\title{
Translational activation by an alternative sigma factor in Bacillus subtilis
}

2

3 Dylan M. McCormick ${ }^{1 \#}$, Jean-Benoît Lalanne ${ }^{1,2,4 \#}$, Tammy C. T. Lan ${ }^{3}$, Silvi Rouskin ${ }^{3}$, Gene-Wei

$5 \quad{ }^{1}$ Department of Biology, Massachusetts Institute of Technology, Cambridge, MA 02139, USA

62 Department of Physics, Massachusetts Institute of Technology, Cambridge, MA 02139, USA

$10 \quad$ \#These authors contributed equally

$11{ }^{*}$ Corresponding author: gwli@mit.edu

13 Running Head: Alternative sigma factor activates translation

14

15 Keywords: sigma factor, RNA structure, translation efficiency, B. subtilis, dual induction

16 


\section{ABSTRACT}

25 Sigma factors are an important class of bacterial transcription factors that lend specificity to

26 RNA polymerases by binding to distinct promoter elements for genes in their regulons. Here we

27 show that activation of the general stress sigma factor, $\sigma^{\mathrm{B}}$, in Bacillus subtilis paradoxically leads

28 to dramatic induction of translation for a subset of its regulon genes. These genes are

29 translationally repressed when transcribed by the housekeeping sigma factor, $\sigma^{\mathrm{A}}$, owing to

30 extended RNA secondary structures as determined in vivo using DMS-MaPseq. Transcription

31 from $\sigma^{\mathrm{B}}$-dependent promoters liberates the secondary structures and activates translation, leading

32 to dual induction. Translation efficiencies between $\sigma^{\mathrm{B}}$ - and $\sigma^{\mathrm{A}}$-dependent RNA isoforms can

33 vary by up to 100 -fold, which in multiple cases exceeds the magnitude of transcriptional

34 induction. These results highlight the role of long-range RNA folding in modulating translation

35 and demonstrate that a transcription factor can regulate protein synthesis beyond its effects on

36 transcript levels.

38 INTRODUCTION

39 Transcriptional regulation by sigma factors is a hallmark of bacterial gene expression. Sigma

40 factors bind to the core RNA polymerases, forming holoenzymes that can initiate transcription at

41 sites with well-defined sequences. In B. subtilis, most genes are transcribed by the housekeeping

42 sigma factor $\sigma^{\mathrm{A}}$, and some are additionally or exclusively transcribed by alternative sigma

43 factors that control specific processes such as sporulation and motility (Haldenwang 1995;

44 Helmann 2019). The alternative sigma factor $\sigma^{\mathrm{B}}$ is involved in the general stress response

45 (Haldenwang and Losick 1979; Hecker et al. 2007; Price 2014; Haldenwang 1995) and initiates

46 transcription for over two hundred genes with well-defined promoter sequences (Nicolas et al. 
47 2012; Petersohn et al. 1999; Zhu and Stülke 2018). Induction of transcription leads to corresponding increases in RNA levels (Figure 1A).

Translational regulation is also widespread in B. subtilis, although it is not typically

50 thought to be controlled by transcription factors. Differential translation among genes in the

51 same operon is largely driven by differences in mRNA secondary structure (Burkhardt et al.

52 2017) and is important for stoichiometric production of proteins in the same complex or

53 metabolic pathway (Lalanne et al. 2018; Li et al. 2014). Translation can be additionally regulated

54 by RNA-binding proteins or riboswitches that modulate the accessibility of the ribosome binding

55 sites on the mRNA (Breaker 2018; Yakhnin et al. 2004, 2007). Operons are often controlled both

56 transcriptionally and translationally (Figure 1A), but seldomly by the same regulator (Bastet et

57 al. 2018; Chauvier et al. 2017; Hollands et al. 2012).

Here we show that the transcription factor $\sigma^{\mathrm{B}}$ not only activates transcription, but also

59 derepresses translation for a subset of its regulon genes. Using Rend-seq (end-enriched RNA-

60 seq) (Lalanne et al. 2018) and ribosome profiling, we identified 12 genes whose apparent

61 translation efficiency is increased substantially during $\sigma^{\mathrm{B}}$ activation. Most of them are

62 transcribed from a $\sigma^{\mathrm{B}}$-dependent promoter as well as at least one $\sigma^{\mathrm{A}}$-dependent promoter,

63 generating multiple transcript isoforms. By modulating $\sigma^{\mathrm{B}}$ activities, we found that each

64 transcript isoform is associated with a distinct translation efficiency, with strongly repressed

65 translation for $\sigma^{\mathrm{A}}$-driven isoforms and elevated translation for $\sigma^{\mathrm{B}}$-driven isoforms. These were

66 orthogonally confirmed using a fluorescent reporter in a subset of examples. Both computational

67 RNA folding and in vivo structural probing by DMS-MaPseq (Zubradt et al. 2016) indicate that

68 the repressed $\sigma^{\mathrm{A}}$-driven isoforms possess extended RNA secondary structures that sequester the

69 ribosome binding sites. On the other hand, $\sigma^{\mathrm{B}}$-driven isoforms have shorter 5' UTRs that only 
70 include the regions corresponding to the second halves of the extended stem-loops in the longer

$71 \sigma^{\mathrm{A}}$-driven isoforms. Therefore, $\sigma^{\mathrm{B}}$ can simultaneously activate both transcription and translation

72 by modulating isoform-specific secondary structures.

\section{RESULTS}

\section{$\sigma^{B}$ activates translation for a subset of its regulon}

76 We first observed translational activation of $\sigma^{\mathrm{B}}$ regulon genes while profiling gene expression

77 for a $B$. subtilis strain with an elevated general stress response during steady-state growth due to

78 a genetic modification (Methods). Rend-seq and ribosome profiling data were generated to

79 quantify the mRNA levels and protein synthesis rates, respectively, for both the wild type (" $\sigma^{\mathrm{B}}$

80 inactive") and the genetically modified strain (" $\sigma^{\mathrm{B}}$ active"). The density of ribosome footprints

81 of a gene provides an estimate for the relative rate of protein synthesis, provided that most

82 ribosomes complete translation to yield full-length polypeptides and that the elongation time

83 averaged across the entire transcript is constant (Ingolia et al. 2009; Li 2015; Lalanne et al. 2018;

84 Li et al. 2014). Translation efficiency (TE), defined as the rate of protein production per mRNA

85 molecule, can then be estimated from Rend-seq and ribosome profiling data by calculating the

86 per-gene ribosome profiling coverage over Rend-seq coverage, i.e., the ribosome density along a

87 transcript (Li 2015; Li et al. 2014). Given $\sigma^{\mathrm{B}}$ 's well-understood role in transcription initiation,

88 we expected its regulon members to change in mRNA levels and not TE.

90 in protein synthesis rate (ribosome profiling) than in mRNA levels (Rend-seq). Between the two

91 conditions, $25 \%$ of the annotated $\sigma^{\mathrm{B}}$ regulon genes (Zhu and Stülke 2018) had substantially

92 different expression levels (56/225 with $>3.7$-fold change, Figure 1B and 1C). Although most 
93 genes showed concordant changes in mRNA levels and protein synthesis rates, a notable

94 population $(21 \%, 12 / 56)$ exhibited a considerably greater increase in protein synthesis rates than

95 mRNA levels ( $>2.7$-fold), suggesting an increase in apparent translation efficiency (Figure 1D).

96 Among these translationally activated $\sigma^{\mathrm{B}}$ regulon genes, the magnitude of TE increases often

97 exceeded the rise in mRNA levels, as most genes $(75 \%, 9 / 12)$ exhibited a fold change in

98 apparent TE accounting for $>50 \%$ of the observed fold change in protein synthesis rate (Figure

99 1E). Hence, translational induction contributes to the majority of the increase in expression of a

100 subset of the $\sigma^{\mathrm{B}}$ regulon, suggesting a yet-unknown strategy for activating translation following

$101 \quad \sigma^{\mathrm{B}}$ induction.

102

$103 \sigma^{\mathbf{B}}$-dependent alternative mRNA isoforms drive translational upregulation

104 To identify the regulatory features that could drive translational upregulation, we examined the

105 transcript architecture of translationally activated $\sigma^{\mathrm{B}}$ regulon genes using Rend-seq. Through

106 sparse fragmentation of input RNAs, Rend-seq enriches for the 5' and 3' boundaries of

107 transcripts, enabling the detection and quantification of mRNA isoforms within operons (Lalanne

108 et al. 2018). We observed that the translationally activated $\sigma^{\mathrm{B}}$ regulon genes were found in two

109 or more different RNA isoforms (Figure 2, Figure S1, Figure S2). In particular, 8 of the 12 genes

110 shared a common operon architecture (Figure 2, Figure S1): They were each transcribed both as

111 a part of a polycistronic mRNA from a vegetative $\left(\sigma^{\mathrm{A}}\right.$-dependent $)$ promoter, as well as from their

112 own $\sigma^{\mathrm{B}}$-dependent promoter. As illustrated by the representative genes $c t c$ and $y v r E$, in the

113 absence of stress, the primary isoform was the long, $\sigma^{\mathrm{A}}$-dependent polycistronic mRNA (Figure

114 2). In these transcripts, the ribosome footprint density for ctc and $y v r E$ was much lower

115 compared to their co-transcribed upstream genes. Under $\sigma^{\mathrm{B}}$ induction, additional 5' ends 
116 appeared directly upstream of their coding sequences (Figure 2, red arrows), consistent with the

117 creation of alternative mRNA isoforms from $\sigma^{\mathrm{B}}$-dependent transcription start sites (TSSs, Figure

1182 inset). Furthermore, these additional 5' ends coincide with a sharp increase in ribosome

119 footprint density over the gene bodies.

We found that the short, $\sigma^{\mathrm{B}}$-dependent isoforms of the translationally activated genes had

121 significantly elevated translation efficiency compared to the corresponding long, $\sigma^{\mathrm{A}}$-dependent

122 isoforms. By estimating the relative prevalence of short and long isoforms across Rend-seq and

123 ribosome profiling datasets with different levels of $\sigma^{\mathrm{B}}$ induction, we could infer the individual

124 translation efficiency for each isoform (Figure 3A, Figure S3, Methods), hereafter referred to as

125 the isoform-specific translation efficiency. Compared to the $\sigma^{\mathrm{A}}$-dependent isoforms, we found

126 that the TE for the $\sigma^{\mathrm{B}}$ isoform was 3- to 100 -fold larger (median $=8.4$, Figure $\left.3 \mathrm{C}\right)$. The $\sigma^{\mathrm{A}}$

127 isoform-specific TEs were all below the median TE across the transcriptome $(5 / 8$ in the bottom

128 quartile, Figure 3B), whereas the $\sigma^{\mathrm{B}}$ isoform-specific TEs were all above the median (7/8 in the

129 top quartile). These results indicate that these $\sigma^{\mathrm{A}}$-dependent isoforms are translationally

130 repressed compared to most genes, whereas the $\sigma^{\mathrm{B}}$-dependent isoforms are translationally

131 activated.

132 We confirmed that TE was isoform-specific using fluorescent reporter constructs for $c t c$

133 and $y v r E$ (Figure 3D). Specifically, we fused the fluorescent protein $\mathrm{mNeonGreen}$ to the C-

134 terminal end of each gene. For each fusion protein ( $c t c-m N e o n, y v r E-m N e o n)$, two distinct

135 isoform-specific 5' untranslated region (5' UTR) variants were placed under the control of an

136 ectopic promoter: 1) a short-isoform variant (S) that included each gene's native 5' UTR

137 corresponding to the $\sigma^{\mathrm{B}}$-dependent isoform (as identified by Rend-seq), and 2) a long-isoform

138 variant (L) that included $\sim 100$ additional nucleotides in the upstream region, which covers a 
139 portion of the coding sequence (CDS) of the upstream gene in the operon. Additionally, a start

140 codon and non-native ribosome binding site (RBS) were inserted directly upstream to enable

141 translation of the truncated upstream CDS in the long-isoform variant. We then quantified the

142 isoform-specific TE for each construct by normalizing relative protein expression (determined

143 from fluorescence, Methods) to relative mRNA levels (from RT-qPCR, Methods). We found that

144 these isoform-specific TEs qualitatively recapitulated our sequencing-based measurements

145 (Figure 3D). Specifically, the isoform-specific TE of the long-isoform constructs was roughly 4-

146 to 6-fold lower than that of the short-isoform constructs, although any further decreases were

147 difficult to quantify due to high background fluorescence. Nevertheless, inclusion of upstream

148 sequence elements was sufficient to produce a large reduction in TE in the absence of the general

149 stress response, which suggests that features in the $\sigma^{\mathrm{A}}$-dependent isoforms can repress translation

150 of the downstream $\sigma^{\mathrm{B}}$ regulon gene. Given the many functions that RNA secondary structure

151 plays in shaping translation in bacteria (Bhattacharyya et al. 2018; Boël et al. 2016; Borujeni et

152 al. 2017; Cambray et al. 2018; Chiaruttini and Guillier 2020; Espah Borujeni and Salis 2016;

153 Goodman et al. 2013; Kudla et al. 2009; Lodish 1968; Li et al. 2014), we aimed to determine if

154 structures in the $\sigma^{\mathrm{A}}$-dependent isoforms could explain the observed impact on translation.

156 Extensive secondary structure is associated with translationally repressed, $\sigma^{\mathrm{A}}$-dependent

157 isoforms

158 To understand the possible role of mRNA secondary structures in setting isoform-specific

159 translation efficiency, we computationally folded for the $\sigma^{\mathrm{A}}$-dependent isoforms of $c t c$ and $y v r E$.

160 By mapping the putative Shine-Dalgarno (SD) sequences that recruit ribosome binding (Shine

161 and Dalgarno 1974), onto minimum free energy (MFE) structures (Methods), we found that the 
162 majority of bases in the SD sequences were sequestered deep in stable, long-range structures

163 (Figure 4A). Strikingly, in both cases the $\sigma^{\mathrm{B}}$-dependent 5' ends were located inside the loop of

164 the long RNA stems, such that the short, $\sigma^{\mathrm{B}}$-generated isoforms have their 5' UTRs entirely

165 liberated from these extended secondary structures. The likelihood of SD sequestration was

166 further supported by calculating the base pairing probability for each position in the SD

167 sequences, which revealed that the majority of positions were predicted to be paired across the

168 full thermodynamic ensemble (base-pairing probability $\approx 1$ ). Given that SD sequences facilitate

169 ribosome recruitment to mRNA to initiate translation, we expected that the presence of extensive

170 secondary structure at and around these elements in the $\sigma^{\mathrm{A}}$-dependent isoforms could plausibly

171 repress translation of the downstream $\sigma^{\mathrm{B}}$ regulon gene. However, numerous factors in the

172 cellular microenvironment affect the folding dynamics of RNAs, yielding in vivo structures that

173 can differ substantially from their in silico counterparts (Mustoe et al. 2018; Rouskin et al. 2014;

174 Spitale et al. 2015; Burkhardt et al. 2017). Accordingly, we decided to experimentally validate

175 these computationally predicted structures for the $\sigma^{\mathrm{A}}$-dependent isoforms of $c t c$ and $y v r E$.

176 We employed the RNA structure probing method DMS-MaPseq to quantify mRNA

177 structures in vivo. This technique involves treating RNA with the methylating agent dimethyl

178 sulfate (DMS) to modify the base-pairing faces of accessible adenine and cytosine nucleobases.

179 These modifications are subsequently encoded as mutations during reverse transcription using a

180 specialized thermostable group II intron reverse transcriptase, generating a mutational signal that

181 is detectable using high-throughput sequencing and has been shown to correlate with base

182 accessibility (Tomezsko et al. 2020; Zubradt et al. 2016). We used a targeted version of DMS-

183 MaPseq to specifically reverse transcribe and amplify the predicted structural region in the $\sigma^{\mathrm{A}}$ -

184 dependent isoforms of $c t c$ and $y v r E$ following DMS treatment in vivo (Figure 4B). After 
185 sequencing these amplicons, we examined the per-base mutational fractions against a control without DMS treatment and confirmed that DMS induced a characteristic signal at amino bases

187 (Figure 4C).

189 (Methods) and found strong agreement with the earlier MFE structures (Figure 4D). In

190 particular, the regions containing the SD sequences were indeed highly structured in vivo and

191 thus less accessible to the translation machinery. Additionally, these structured regions were

192 robust to the folding window size (Methods). These extended structures that occlude the

193 ribosome binding sites are consistent with the repressed translation of the long, $\sigma^{\mathrm{A}}$-dependent

194 isoforms.

After validating the computationally predicted secondary structures by DMS-MaPseq, we extended our computational analysis to additional translationally activated $\sigma^{\mathrm{B}}$ regulon genes and

197 found a consistent pattern of characteristic structures in the $\sigma^{\mathrm{A}}$-dependent isoforms that sequester

198 the sequence elements required for translation initiation (Figure 5). Similar to $c t c$ and $y v r E$, the

199 remaining 6 genes for which we estimated isoform-specific TE all displayed MFE structures

200 with the SD sequences located in extended stem-loops, and base pairing probabilities indicated

201 that the SD sequences were predominantly paired. These results suggest that these other $\sigma^{\mathrm{A}}$ -

202 dependent long isoforms are also translationally repressed by extensive secondary structures, like

203 the orthogonally validated instances of $c t c$ and $y v r E$.

\section{Internal $\sigma^{\mathbf{B}}$ promoters liberate mRNA secondary structure and activate translation}

206 In contrast to being repressed in the $\sigma^{\mathrm{A}}$-dependent isoforms, genes in the short, $\sigma^{\mathrm{B}}$-dependent

207 isoforms had above-normal levels of translation (Figure 3C). The single-nucleotide resolution 
208 afforded by Rend-seq data revealed a common feature among this group of genes: the TSSs of

209 the $\sigma^{\mathrm{B}}$-dependent isoforms were located within the extended secondary structure, often inside the

210 loop region or in the downstream stem (Figure 4A, Figure 5, magenta and arrow). Therefore, $\sigma^{\mathrm{B}}-$

211 driven transcription generates isoforms with 5' UTRs that lack the upstream portion of the stem

212 sequestering the $\mathrm{SD}$ sequence in the long, $\sigma^{\mathrm{A}}$-dependent isoforms, thereby freeing up the

213 ribosome binding site for efficient translation initiation.

214 The prevalence of this regulation suggests an alternative configuration for $\sigma^{\mathrm{B}}$-dependent

215 gene expression that does not entirely rely on its canonical role as acting at the transcriptional

216 level. In this operonic architecture, $\sigma^{\mathrm{A}}$-driven promoters produce long, polycistronic mRNAs

217 containing stable structures that impede translation initiation for $\sigma^{\mathrm{B}}$ regulon genes located at the

218 ends of these transcripts (Figure 6). When activated by stress, however, $\sigma^{\mathrm{B}}$ initiates transcription

219 from alternative promoters directly upstream of its regulon genes, bypassing the inhibitory

220 secondary structures and thereby promoting ribosome binding on these shorter mRNAs. The

221 resulting increase in protein expression predominantly arises from a greater ribosome flux on

222 these transcripts, demonstrating a novel function for $\sigma^{\mathrm{B}}$ in regulating gene expression in a

223 simultaneous transcriptional-translational induction.

225 DISCUSSION

226 Bacterial sigma factors have long been studied as quintessential examples of gene regulation.

227 Mechanistically, their direct effects on transcription initiation are well-understood (Paget 2015).

228 We expand this view by demonstrating that the alternative sigma factor $\sigma^{\mathrm{B}}$ in $B$. subtilis can also

229 influence translation initiation for several of its regulon genes. Translation activation is

230 accomplished by modulating isoform-specific RNA secondary structures that normally impede 
231 translation initiation. This multifunctional control of transcription and translation by a single

232 trans-acting factor serves as a strategy to enable massive upregulation of gene expression under

233 specific cellular conditions.

234 The RNA secondary structures that impede translation in the long, $\sigma^{\mathrm{A}}$-dependent

235 isoforms often include regions of the upstream open reading frames (ORFs), raising questions

236 about whether ribosomes translating the upstream ORFs may perturb the formation of the

237 inhibitory secondary structures. Ribosomes are known to unwind structured regions of RNA as

238 they elongate over coding sequences (Takyar et al. 2005; Wen et al. 2008). We observed that the

239 stop codon of the upstream gene in the operon was typically located within the large stem-loop

240 (Figure 4, Figure 5). This places ribosomes in proximity to the critical structural elements if the

241 upstream message is actively translated. However, the results from our fluorescent reporter assay

242 show that this configuration is not capable of fully restoring translation for either ctc or $y v r E$,

243 despite the upstream gene being driven by an exogenous ribosome binding site with the

244 consensus SD sequence. These data suggest that translation of the upstream gene is insufficient

245 to fully derepress downstream genes, presumably because the ribosome footprint does not extend

246 sufficiently downstream to disrupt RNA structure, or possibly due to rapid refolding of

247 secondary structures after ribosomes pass through.

248 What is the utility of this regulatory strategy? From an evolutionary perspective, it seems

249 counterintuitive for these genes to be found within larger operons despite being lowly translated.

250 We could instead imagine a transcription terminator evolving in the region between the upstream

251 genes in the operon and the $\sigma^{\mathrm{B}}$-dependent TSS, which would ensure that the $\sigma^{\mathrm{B}}$ regulon gene is

252 only induced upon activation of the general stress response. One potential explanation for

253 multifunctional regulation is to allow fine-tuned expression of some $\sigma^{\mathrm{B}}$ regulon genes during 
254 non-stress conditions. On the one hand, this transcript architecture enables these genes to be

255 transcribed during exponential growth. On the other hand, translation may have been selected

256 against in the same condition to avoid fitness defects from overexpression. In this case, the

257 observed basal expression from the $\sigma^{\mathrm{A}}$-driven isoforms would be sufficient for their functions

258 during non-stress conditions.

259 Another possible explanation for this regulatory strategy could be that small amounts of

260 these proteins are necessary for coping with general stress during transitional periods where $\sigma^{\mathrm{B}}$

261 has already been activated but synthesis of general stress proteins is still ongoing. A fitness

262 benefit would be challenging to identify except in specific conditions where the cell relies on one

263 of these particular $\sigma^{\mathrm{B}}$ regulon genes for survival. Indeed, extensive phenotyping of $\sigma^{\mathrm{B}}$-regulon

264 member deletions under varied stresses has demonstrated the limited impact of individual

265 proteins on cell fitness (Höper et al. 2005). Identifying the exact stress conditions in which this

266 regulatory strategy confers a fitness advantage constitutes an interesting future direction.

268 characterization of sigma factor-mediated dual induction (Figure 6) expands our view of the

269 regulatory roles of sigma factors and reveals an intriguing principle of bacterial genome

270 organization that could be further investigated in similar organisms. 


\section{MATERIALS AND METHODS}

\section{Strains and strain construction}

279 Strains used to generate new data in this study are listed in Table 1. Strains pertaining to matched

280 Rend-seq and ribosome profiling datasets (from GEO accession GSE162169) are listed in Table

281 S1.

To construct the strains for the fluorescent reporter assay, the genes $c t c$ and $y v r E$ (with

283 variable upstream regions) were fused to the fluorescent protein $\mathrm{mNeonGreen} \mathrm{with} \mathrm{a} \mathrm{C-terminal}$

284 linker and cloned into pJBL044 under the constitutive promoter Pveg using Gibson assembly

285 (New England Biolabs). The original pJBL044 plasmid was constructed using isothermal

286 assembly from a fragment of pDR160 (Bose and Grossman 2011), a kanR cassette (Guérout-

287 Fleury et al. 1995), levB homology regions, the Pveg promoter, and the strong efp terminator.

288 The assembled plasmids were transformed into Mix and Go! E. coli DH5 Alpha Competent Cells

289 (Zymo Research) per the manufacturer's instructions and isolated using a QIAprep Spin

290 Miniprep Kit (QIAGEN). The fusion constructs were then integrated into BS168 at the levB

291 locus using standard cloning techniques (Harwood, C R and Cutting 1990), and successful

292 recombinants were verified by colony PCR. All plasmids and recombinants (see Table 1) were

293 further validated by Sanger sequencing (Quintara Biosciences).

\section{Growth conditions}

296 Unless indicated otherwise, all strains were grown at $37^{\circ} \mathrm{C}$ with shaking $(250 \mathrm{rpm})$ in $\mathrm{LB}$

297 supplemented with carbenicillin $(100 \mu \mathrm{g} / \mathrm{mL}$ for E. coli $)$ and/or kanamycin $(50 \mu \mathrm{g} / \mathrm{mL}$ for E. coli,

$2985 \mu \mathrm{g} / \mathrm{mL}$ for B. subtilis) when appropriate. For overnight cultures, LB liquid media was

299 inoculated with single colonies from LB agar plates. 
For matched Rend-seq/ribosome profiling datasets, strains were grown in LB or

conditioned MCC medium (Parker et al. 2020; Lalanne et al. 2020) with various inducer (xylose,

IPTG) concentrations (see Table S1). For these datasets, cells were grown in exponential phase

303 for at least 10 doublings before harvesting at $\mathrm{OD}_{600} \approx 0.3$.

305 Existing Rend-seq and ribosome profiling datasets

306 Matched Rend-seq and ribosome profiling datasets used to identify genes with increased TE

307 (Figure 1) and to estimate the short isoform fraction and corresponding apparent TE (Figure 3,

308 Figure S3) were obtained from GEO accession GSE162169 (Lalanne et al. 2020). These datasets

309 display a range of $\sigma^{\mathrm{B}}$ activation due to a diverse set of genetic modifications and growth media.

310 In particular, we previously identified that tuning the expression of translation termination

311 factors RF2 and PrmC activate $\sigma^{\mathrm{B}}$ to varying degrees (Lalanne et al. 2020). For example, the $\sigma^{\mathrm{B}}$

312 active data presented in Figures 1 and 2 correspond to a CRISPRi knockdown of RF2, while $\sigma^{\mathrm{B}}$

313 inactive corresponds to wild-type. Importantly, although it is possible that different RF2 levels

314 could affect translation initiation (and therefore TE) of genes (Lalanne et al. 2020), none of the

315 genes that show a substantial increase in TE (Figure 1) have a UGA stop codon or are co-

316 transcribed with a gene ending with UGA stop (UGA being the stop codon cognate to RF2).

317 Hence, the molecular causes of $\sigma^{\mathrm{B}}$ activation are distinct and independent from the mechanisms

318 leading to translational activation characterized here.

320 Quantification of mRNA level, ribosome footprint density, and translation efficiency

321 From pile-up files (.wig format), the mRNA level corresponding to a gene was quantified as the $3221 \%$ winsorized average read density for 3'-end mapped Rend-seq reads across the body of the 
323 gene, excluding a $40 \mathrm{nt}$ region the start and end of the gene (start $+40 \mathrm{nt}$ to end $-40 \mathrm{nt}$ for

324 averaging). Ribosome footprint read density was similarly calculated (1\% winsorized density

325 from start+40 nt to end-40 nt). Read densities were then normalized to rpkm (reads per kilobase

326 per million reads mapped) using the total number of reads mapping to non-rRNA or tRNAs. For

327 all genes, bootstrap (randomly sampling from the distribution of read counts per position across

328 the body of the gene and calculating the corresponding resampled density and downstream

329 quantities) was used as a measure of technical and read count variability. Error bars in Figures

$330 \quad 3 \mathrm{~A}$ and $\mathrm{S} 3$ correspond to the standard deviation across bootstrap subsamplings. Large error bars

331 correspond to large counting noise (regions with few reads mapped). The translation efficiency

332 of each gene was calculated as the ribosome profiling rpkm divided by the Rend-seq rpkm. Only

333 genes with $>50$ reads mapped were considered to identify candidates with substantially elevated

334 TE (Figure 1).

Determination of isoform-specific TE

337 To estimate the isoform-specific TE for particular genes, we assume that each individual mRNA

338 isoform has a distinct TE, and that the total ribosome footprint density for a gene with multiple

339 mRNA isoforms is equal to the sum of the isoform-specific TEs weighted by the mRNA

340 abundance of each isoform.

341 Specifically, consider a two-gene operon with a long isoform that includes both gene 1

342 and gene 2 as well as a short isoform that contains gene 2 exclusively (schematically illustrated

343 in Figure S3A). Denote overall mRNA level for genes 1 and 2 by $m_{1}$ and $m_{2}$, and overall

344 ribosome footprint density $r_{1}$ and $r_{2}$ for the two genes respectively. Further, let $m_{\text {short }}, m_{\text {long }}$

345 be the level of the short and long isoform respectively, and $T E^{2, \text { short }}, T E^{2, l o n g}$ the 
corresponding isoform-specific TE. Note that the overall mRNA level for genes 1 and 2 are related to isoform mRNA levels by: $m_{1}=m_{\text {long }}$ and $m_{2}=m_{\text {short }}+m_{\text {long }}$. Hence, from the

348 total mRNA level for both genes, we can infer the isoform mRNA levels: $m_{\text {long }}=m_{1}$, and $m_{\text {short }}=m_{2}-m_{1}$.

354 for gene 2 as $f_{\text {short }}:=\frac{m_{\text {short }}}{m_{2}}=\frac{m_{2}-m_{1}}{m_{2}}$. We note that for genes in conditions with little to no short isoform expression, the estimated short isoform fraction may be negative as a result of the technical variability in coverage. 
367 footprint densities were calculated as the average read densities across these regions in Rend-seq 368 and ribosome profiling data, respectively.

To determine the uncertainty on estimated isoform-specific TEs, linear regressions were

370 performed on bootstrap resampling estimates for the short isoform fractions and apparent TEs.

371 Each bootstrap regression provided an estimated $\mathrm{TE}^{\text {long }}$ and $\mathrm{TE}^{\text {short }}$. The error bars for these

372 quantities (Figure 3A, Figure 3C, Figure S3B) were taken as the standard deviations of these

373 bootstrap estimates.

$374 \quad$ For the genes that do not belong to the group with the characteristic long, $\sigma^{\mathrm{A}}$-dependent

375 isoforms and short, $\sigma^{\mathrm{B}}$-dependent isoforms (Figure $\mathrm{S} 2$ ), their alternative promoters are too close

376 to allow proper quantification of isoform-specific abundances. These were thus excluded from

377 the above analyses.

\section{Fluorescent reporter assay}

380 For the fluorescence reporter assay, the strains GLB115, GLB572, GLB573, GLB574, and

381 GLB575 were grown to $\mathrm{OD}_{600} \approx 1-2$ and then back-diluted 200-fold into fresh media. Three

382 technical replicates per culture were grown at $37^{\circ} \mathrm{C}$ for $12 \mathrm{hr}$ in a BioTek Synergy $\mathrm{H} 1$ microplate 383 reader, and absorbance $(600 \mathrm{~nm})$ and fluorescence intensity (EX 485/20 nm, EM 520/20 nm)

384 were measured every $5 \mathrm{~min}$. Fluorescence was normalized by absorbance at each time point, and 385 any background signal from cellular/media autofluorescence was removed by subtracting the 386 mean normalized fluorescence values of the wild-type BS168 replicates. These quantities were 387 then converted to relative values by normalizing proportionally to the signal for the $\mathrm{S}$ variants. 
with $5 \mathrm{~mL}$ of chilled methanol, spun down at $4^{\circ} \mathrm{C}$ for $10 \mathrm{~min}$, and frozen at $-80^{\circ} \mathrm{C}$ after removing

391 the supernatant. Thawed cell pellets were treated with $100 \mu \mathrm{L}$ of $10 \mathrm{mg} / \mathrm{mL}$ lysozyme in TE, and

392 total RNA was extracted using a RNeasy Mini Kit (QIAGEN). DNA was removed using

393 TURBO DNase (Thermo Fisher Scientific), and RNA was purified using isopropanol

394 precipitation. Reverse transcription was performed using Random Hexamer Primer (Thermo

395 Fisher Scientific) and M-MuLV Reverse Transcriptase (New England Biolabs) per the

396 manufacturer's instructions. RNA levels were measured on a Roche LightCycler 480 Real-Time

397 PCR system using two primer sets for $m$ Neon and one primer set each for the loading controls

398 gyrA and $\operatorname{sig} A(m N e o n \mathrm{~F} 1$, mNeon $\mathrm{R} 1$, mNeon $\mathrm{F} 2$, mNeon $\mathrm{R} 2, \operatorname{gyr} A \mathrm{~F}, \operatorname{gyr} A \mathrm{R}, \operatorname{sig} A \mathrm{~F}, \operatorname{sig} A \mathrm{R}$,

399 see Table 2). The fold change in mNeon RNA levels relative to the $\mathrm{S}$ strains was calculated by

400 taking the average of three technical replicates across each combination of primer sets

401 (mNeon1/gyrA, mNeon1/sigA, mNeon2/gyrA, mNeon2/sigA).

402 Isoform-specific TE was subsequently calculated by normalizing mean relative

403 fluorescence by mean fold change in mNeon RNA levels, and the standard deviation was

404 propagated from each measurement type.

405

\section{RNA secondary structure prediction}

407 Minimum free energy (MFE) structures were predicted using the RNAfold program of the

408 ViennaRNA Package (Lorenz et al. 2011) with default parameters. Base-pairing probabilities

409 were determined by constraining each position in a sequence individually as unpaired and then

410 calculating the partition function from the ensemble free energy computed by RNAfold. The

411 probability of each position being unpaired was calculated by dividing the partition function for

412 the constrained sequence by the partition function for an unconstrained sequence, and the base 
413 pairing probabilities were simply the probabilities of the complements. Putative Shine-Dalgarno

414 (SD) sequences were identified as the region upstream of the start codon that forms the strongest

415 duplex with the anti-Shine-Dalgarno (aSD, 5'-TCACCTCCT-3') sequence in the 16S ribosomal

416 RNA. RNA secondary structures determined using RNAfold were visualized using VARNA

417 v3.93 (Visualization Applet for RNA) (Darty et al. 2009). The structures sequestering the

418 ribosome binding sites shown in Figures 4 and 5 were confirmed to be robust to the specific

419 regions computationally folded, both at the level of secondary structure and base-pairing

420 probabilities of the SD sequences.

421

422 DMS-MaPseq

423 In vivo DMS treatment was performed as previously described (Burkhardt et al. 2017; Zubradt et

424 al. 2016). Specifically, an overnight culture of BS168 was split two ways and back-diluted to

$425 \mathrm{OD}_{600} \approx 2 \times 10^{-4}$. Following regrowth to $\mathrm{OD}_{600} \approx 0.2,15 \mathrm{~mL}$ of each culture was incubated at $37^{\circ} \mathrm{C}$

426 for 2 min with shaking (1000 rpm) after treating one with $750 \mu \mathrm{L}$ of dimethyl sulfate (DMS,

$427 \sim 5 \%$ final concentration). The reaction was stopped by adding $30 \mathrm{~mL}$ of chilled stop solution

428 (30\% $\beta$-mercaptoethanol, 25\% isoamyl alcohol) to each sample, after which they were

429 immediately transferred to ice and spun down at $4^{\circ} \mathrm{C}$ for $8 \mathrm{~min}$. The cell pellets were washed

430 with $8 \mathrm{~mL}$ of chilled wash solution (30\% $\beta$-mercaptoethanol), resuspended in residual wash

431 solution, and frozen at $-80^{\circ} \mathrm{C}$. Thawed cell pellets were treated with $100 \mu \mathrm{L}$ of $10 \mathrm{mg} / \mathrm{mL}$

432 lysozyme in TE, and total RNA lysis buffer (10 mM EDTA, $50 \mathrm{mM}$ sodium acetate) was added

433 to $650 \mu \mathrm{L}$. Total RNA was extracted using hot acid-phenol:chloroform and isopropanol

434 precipitation. 

were again followed. DNA was removed using TURBO DNase, and RNA >200 nt was purified

437 using an RNA Clean \& Concentrator-5 Kit per the manufacturer's instructions (Zymo Research).

438 Ribosomal RNA was depleted using a MICROBExpress Bacterial mRNA Enrichment Kit

439 (Thermo Fisher Scientific), and RNA > $200 \mathrm{nt}$ was again purified using an RNA Clean \&

440 Concentrator-5 Kit. Reverse transcription was performed at $64^{\circ} \mathrm{C}$ for 90 min using $70 \mathrm{ng}$ of RNA

441 from each sample and TGIRT-III (Ingex). The RT primers were specific to each gene (ctc R,

$442 y v r E$ R, see Table 2). The RT reaction was treated with $1 \mu 1$ RNase H (New England Biolabs)

443 and incubated at $37^{\circ} \mathrm{C}$ for $20 \mathrm{~min}$ to remove RNA. Roughly $1 / 10$ of the resulting volume was

444 used as template for a two-step PCR amplification with Phusion High-Fidelity DNA Polymerase

445 (New England Biolabs) per the manufacturer's specifications, which was run for 15-25 cycles

446 with the RT primer serving as the reverse primer (ctc F, yvrE F, see Table 2). PCR products

447 ( 240-290 bp) were purified by gel extraction on an 8\% TBE polyacrylamide gel (Thermo Fisher

448 Scientific) and isopropanol precipitation. Samples with particularly low dsDNA concentrations

449 (as measured on an Invitrogen Qubit 4 Fluorometer) were reamplified for 7-20 additional cycles

450 and purified in the same manner. After adding adapters via PCR, the libraries were sequenced on

451 an Illumina MiSeq (2 x $250 \mathrm{nt}$ reads).

452 To determine the DMS signal, FASTQ files were processed and analyzed using the

453 DREEM (Detection of RNA folding Ensembles using Expectation-Maximization clustering)

454 pipeline with the '--fastq' and '--struct' options (Tomezsko et al. 2020). In brief, paired-end

455 reads were filtered for quality and trimmed using FASTQC v.0.11.8 and TrimGalore 0.4.1,

456 respectively. Reads were aligned to target sequences in the reference genome NC_000964.3 from

457 the NCBI RefSeq database using Bowtie2 2.3.4.1 with the options '--local --no-unal --no- 
458 discordant --no-mixed -X 1000 -L 12'. Mapped reads were represented as bit vectors and

459 clustered by their mutational signatures using the DREEM algorithm with standard parameters

460 (Tomezsko et al. 2020). Per-base mutational fractions were initially quantified using the

461 population-average fraction of mismatches and deletions. Following expectation-maximization

462 (EM) clustering, the DMS reactivity was taken as the mutation rates of the bases in the cluster

$463 \mathrm{~K}=1$. After normalizing to the median of the top $5 \%$ of positions (with the upper limit set to 1.0 ),

464 the DMS signal was used as a folding constraint for predicting RNA secondary structures with

465 the program RNAstructure v.6.0.1 (Reuter and Mathews 2010) Additionally, the folding

466 windows were expanded symmetrically by $50,100,150$, and 200 -nt in either direction to assess

467 the robustness of the predicted folds. RNA secondary structures were again visualized using

468 VARNA v3.93 (Darty et al. 2009). The sequencing datasets for DMS-MaPseq are available

469 online using the GEO accession GSE168393.

470

471 SUPPLEMENTAL MATERIAL

472 Supplemental material (Figures S1-S3, Table S1) is available for this article.

473

\section{ACKNOWLEDGEMENTS}

475 We thank members of the Li lab and the Rouskin lab for critical discussions. We thank S.

476 McKeithen-Mead for providing the mNeon template. This research was supported by NIH grant

477 R35GM124732, the NSF CAREER Award, the Smith Odyssey Award, the Pew Biomedical

478 Scholars Program, a Sloan Research Fellowship, the Searle Scholars Program, the Smith Family

479 Award for Excellence in Biomedical Research; NSERC doctoral Fellowship and HHMI

480 International Student Research Fellowship (to J.-B.L.). 


\section{REFERENCES}

482

483

484

485

486

487

488

Bastet L, Turcotte P, Wade JT, Lafontaine DA. 2018. Maestro of regulation: Riboswitches orchestrate gene expression at the levels of translation, transcription and mRNA decay. RNA Biol.

Bhattacharyya S, Jacobs WM, Adkar B V., Yan J, Zhang W, Shakhnovich EI. 2018. Accessibility of the Shine-Dalgarno Sequence Dictates N-Terminal Codon Bias in E. coli. Mol Cell 70.

Boël G, Letso R, Neely H, Price N, Wong K, Su M, Luff JD, Valecha M, Hunt JF, Everett JK, et al. 2016. Codon influence on protein expression in E . coli correlates with mRNA levels. Nature. http://dx.doi.org/10.1038/nature16509.

Borujeni AE, Cetnar D, Farasat I, Smith A, Lundgren N, Salis HM. 2017. Precise quantification of translation inhibition by mRNA structures that overlap with the ribosomal footprint in Nterminal coding sequences. Nucleic Acids Res 45.

Bose B, Grossman AD. 2011. Regulation of horizontal gene transfer in Bacillus subtilis by activation of a conserved site-specific protease. J Bacteriol 193: 22-29.

Breaker RR. 2018. Riboswitches and translation control. Cold Spring Harb Perspect Biol.

Burkhardt DH, Rouskin S, Zhang Y, Li GW, Weissman JS, Gross CA. 2017. Operon mRNAs are organized into ORF-centric structures that predict translation efficiency. Elife.

Cambray G, Guimaraes JC, Arkin AP. 2018. Evaluation of 244,000 synthetic sequences reveals design principles to optimize translation in escherichia coli. Nat Biotechnol 36: 1005.

Chauvier A, Picard-Jean F, Berger-Dancause JC, Bastet L, Naghdi MR, Dubé A, Turcotte P, Perreault J, Lafontaine DA. 2017. Transcriptional pausing at the translation start site operates as a critical checkpoint for riboswitch regulation. Nat Commun. 
504 Chiaruttini C, Guillier M. 2020. On the role of mRNA secondary structure in bacterial translation. Wiley Interdiscip Rev RNA 11.

Darty K, Denise A, Ponty Y. 2009. VARNA: Interactive drawing and editing of the RNA secondary structure. Bioinformatics. via a Ribosome Drafting Mechanism. J Am Chem Soc jacs.6b01453.

511 Goodman DB, Church GM, Kosuri S. 2013. Causes and effects of N-terminal codon bias in bacterial genes. Science (80- ) 342.

513 Guérout-Fleury AM, Shazand K, Frandsen N, Stragier P. 1995. Antibiotic-resistance cassettes 514 for Bacillus subtilis. Gene 167: 335-336.

515 Haldenwang WG. 1995. The sigma factors of Bacillus subtilis. Microbiol Rev.

516 Haldenwang WG, Losick R. 1979. A modified RNA polymerase transcribes a cloned gene under sporulation control in Bacillus subtilis. Nature 282.

518 Harwood, C R and Cutting SM. 1990. Molecular Biological methods for Bacillus. John Wiley.

519 Hecker M, Pané-Farré J, Uwe V. 2007. SigB-Dependent General Stress Response in Bacillus

520 subtilis and Related Gram-Positive Bacteria. Annu Rev Microbiol 61: 215-236.

521 http://www.annualreviews.org/doi/10.1146/annurev.micro.61.080706.093445.

522 Helmann JD. 2019. Where to begin? Sigma factors and the selectivity of transcription initiation 523 in bacteria. Mol Microbiol 112.

524 Hollands K, Proshkin S, Sklyarova S, Epshtein V, Mironov A, Nudler E, Groisman EA. 2012. 525 Riboswitch control of Rho-dependent transcription termination. Proc Natl Acad Sci U S A. 526 Höper D, Völker U, Hecker M. 2005. Comprehensive characterization of the contribution of 
individual SigB-dependent general stress genes to stress resistance of Bacillus subtilis. $J$ Bacteriol 187: 2810-2826.

529 Ingolia NT, Ghaemmaghami S, Newman JRS, Weissman JS. 2009. Genome-wide analysis in vivo of translation with nucleotide resolution using ribosome profiling. Science 324: 218 223. http://www.sciencemag.org/content/324/5924/218.full.pdf.

532 Kudla G, Murray AW, Tollervey D, Plotkin JB. 2009. Coding-sequence determinants of expression in escherichia coli. Science (80-) 324.

534 Lalanne J, Parker DJ, Li G. 2020. Spurious regulatory connections dictate the expression-fitness landscape of translation termination factors. bioRxiv 1-25.

Lalanne JB, Taggart JC, Guo MS, Herzel L, Schieler A, Li GW. 2018. Evolutionary Convergence of Pathway-Specific Enzyme Expression Stoichiometry. Cell 749-761.

Li GW. 2015. How do bacteria tune translation efficiency? Curr Opin Microbiol 24.

539 Li GW, Burkhardt D, Gross C, Weissman JS. 2014. Quantifying absolute protein synthesis rates $540 \quad$ reveals principles underlying allocation of cellular resources. Cell 157: 624-635. http://dx.doi.org/10.1016/j.cell.2014.02.033.

542 Lodish HF. 1968. Bacteriophage f2 RNA: Control of translation and gene order. Nature 220.

543 Lorenz R, Tafer H, Höner zu Siederdissen C, Stadler PF, Bernhart SH, Hofacker IL, Flamm C.

544 2011. ViennaRNA Package 2.0. Algorithms Mol Biol 6: 26.

545 Mustoe AM, Busan S, Rice GM, Hajdin CE, Peterson BK, Ruda VM, Kubica N, Nutiu R,

546 Baryza JL, Weeks KM. 2018. Pervasive Regulatory Functions of mRNA Structure

547 Revealed by High-Resolution SHAPE Probing. Cell 173: 181-195.e18.

$548 \quad$ http://dx.doi.org/10.1016/j.cell.2018.02.034.

549 Nicolas P, Mäder U, Dervyn E, Rochat T, Leduc A, Pigeonneau N, Bidnenko E, Marchadier E, 
Hoebeke M, Aymerich S, et al. 2012. Condition-dependent transcriptome reveals high-level regulatory architecture in Bacillus subtilis. Science 335: 1103-6. http://classic.sciencemag.org/content/335/6072/1103.full.

553 Paget MS. 2015. Bacterial sigma factors and anti-sigma factors: Structure, function and 554 distribution. Biomolecules 5.

555 Parker DJ, Lalanne J-B, Kimura S, Johnson GE, Waldor MK, Li G-W. 2020. Growth-Optimized Aminoacyl-tRNA Synthetase Levels Prevent Maximal tRNA Charging. Cell Syst 1-10.

Petersohn A, Bernhardt J, Gerth U, Höper D, Koburger T, Völker U, Hecker M. 1999. directed search and oligonucleotide hybridization. J Bacteriol.

560 Price CW. 2014. General Stress Response in Bacillus subtilis and Related Gram-Positive Bacteria. In Bacterial Stress Responses, pp. 301-318, ASM Press, Washington, DC, USA.

Reuter JS, Mathews DH. 2010. RNAstructure: Software for RNA secondary structure prediction and analysis. BMC Bioinformatics 11.

564 Rouskin S, Zubradt M, Washietl S, Kellis M, Weissman JS. 2014. Genome-wide probing of RNA structure reveals active unfolding of mRNA structures in vivo. Nature 505: 701-5. http://www.ncbi.nlm.nih.gov/pubmed/24336214.

567 Shine J, Dalgarno L. 1974. The 3' terminal sequence of Escherichia coli 16S ribosomal RNA: complementarity to nonsense triplets and ribosome binding sites. Proc Natl Acad Sci U S A 
573 Takyar S, Hickerson RP, Noller HF. 2005. mRNA helicase activity of the ribosome. Cell 120.

574 Tomezsko PJ, Corbin VDA, Gupta P, Swaminathan H, Glasgow M, Persad S, Edwards MD,

575 Mcintosh L, Papenfuss AT, Emery A, et al. 2020. Determination of RNA structural

576 diversity and its role in HIV-1 RNA splicing. Nature.

577 Wen J-D, Lancaster L, Hodges C, Zeri A-C, Yoshimura SH, Noller HF, Bustamante C, Tinoco I.

578 2008. Following translation by single ribosomes one codon at a time. Nature 452: 598-603.

579 http://www.nature.com/nature/journal/v452/n7187/pdf/nature06716.pdf.

580 Yakhnin H, Pandit P, Petty TJ, Baker CS, Romeo T, Babitzke P. 2007. CsrA of Bacillus subtilis regulates translation initiation of the gene encoding the flagellin protein (hag) by blocking ribosome binding. Mol Microbiol.

583 Yakhnin H, Zhang H, Yakhnin A V., Babitzke P. 2004. The trp RNA-Binding Attenuation Protein of Bacillus subtilis Regulates Translation of the Tryptophan Transport Gene trpP (yhaG) by Blocking Ribosome Binding. J Bacteriol.

Zhu B, Stülke J. 2018. SubtiWiki in 2018: From genes and proteins to functional network annotation of the model organism Bacillus subtilis. Nucleic Acids Res 46: D743-D748.

588 Zubradt M, Gupta P, Persad S, Lambowitz AM, Weissman JS, Rouskin S. 2016. DMS-MaPseq

589 for genome-wide or targeted RNA structure probing in vivo. Nat Methods 14: 75-82.

$590 \quad$ http://dx.doi.org/10.1038/nmeth.4057. 
TABLES

TABLE 1. Strains and plasmids used in this study

\begin{tabular}{|l|l|l|}
\hline \multicolumn{1}{|c|}{ Name } & \multicolumn{1}{|c|}{ Genotype } & \multicolumn{1}{|c|}{ Origin } \\
\hline GLB115 & BS168, wild-type Bacillus subtilis subsp. 168 & J. Wang \\
\hline GLB572 & BS168 levB::Pveg-ctc-S-mNeon kanR & This study \\
\hline GLB573 & BS168 levB::Pveg-ctc-L-mNeon kanR & This study \\
\hline GLB574 & BS168 levB::Pveg-yvrE-S-mNeon kanR & This study \\
\hline GLB575 & BS168 levB::Pveg-yvrE-L-mNeon kanR & This study \\
\hline pDMM001 & pJBL044 ctc-S-mNeon & This study \\
\hline pDMM002 & pJBL044 ctc-L-mNeon & This study \\
\hline pDMM003 & pJBL044 yvrE-S-mNeon & This study \\
\hline pDMM004 & pJBL044 yvrE-L-mNeon & This study \\
\hline
\end{tabular}

\section{TABLE 2. Oligos used in this study}

\begin{tabular}{|c|c|}
\hline Name & Sequence (5'-3') \\
\hline$m$ Neon F1 & CGACCCACGAACTGCATATT \\
\hline$m$ Neon R1 & GCCCGTAGTATAGCTCCATTTG \\
\hline$m$ Neon F2 & GAACCCTAACGATGGCTATGAG \\
\hline$m$ Neon R2 & CTCCATTTGAAGGTCGAGATGA \\
\hline$g y r A \mathrm{~F}$ & CTCGATGCAGTTATCTCCCTTATC \\
\hline gyrA $\mathrm{R}$ & TCGCTTGTGCTTGCTTCT \\
\hline $\operatorname{sig} A \mathrm{~F}$ & AGATTGAAGAAGGTGACGAAGAAT \\
\hline sigA R & TCAGATCAAGGAACAGCATACC \\
\hline$c t c \mathrm{R}$ & TGACACAGGTTTGTTACCCGTATCCTTCCC \\
\hline$y v r E \mathrm{R}$ & AGGGTCAAAGATGTGGAGCTCGCTCC \\
\hline$c t c \mathrm{~F}$ & TATCAGGCCCTGCGGTTGAACGGAT \\
\hline$y v r E \mathrm{~F}$ & CCGCTACTACAGAGGGACGAACACAA \\
\hline
\end{tabular}


FIGURES

A
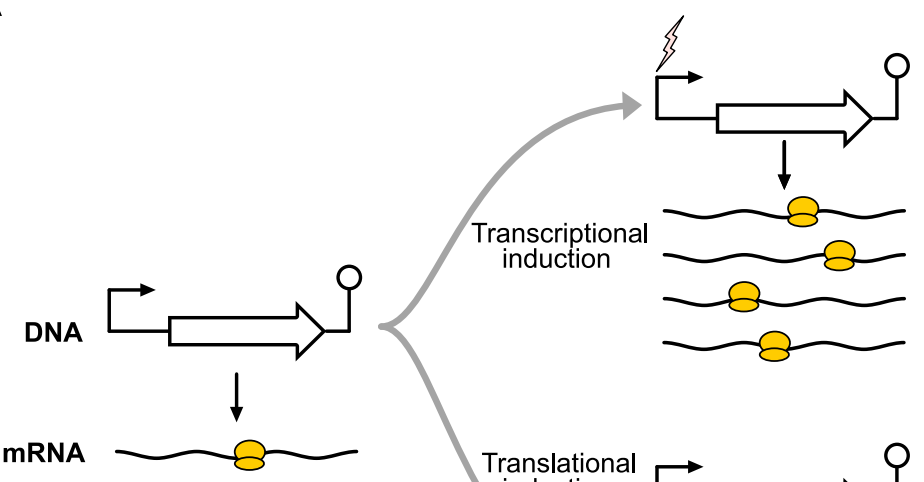

mRNA

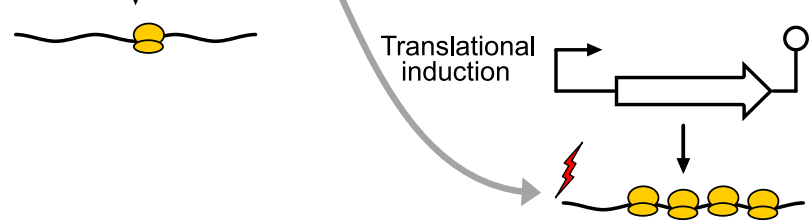

$E$

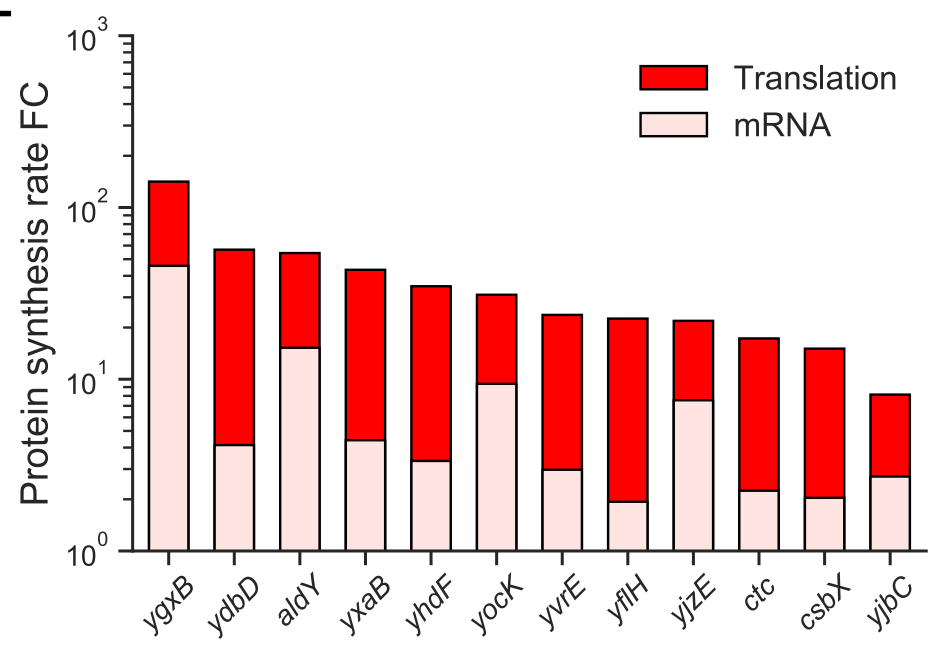

B

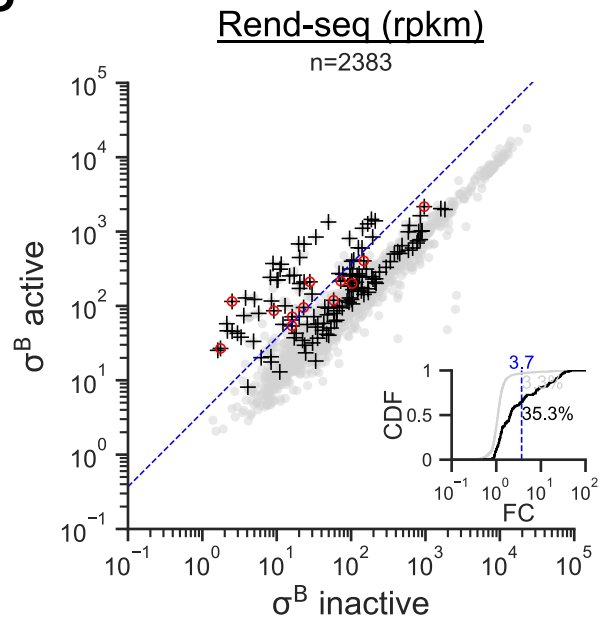

C

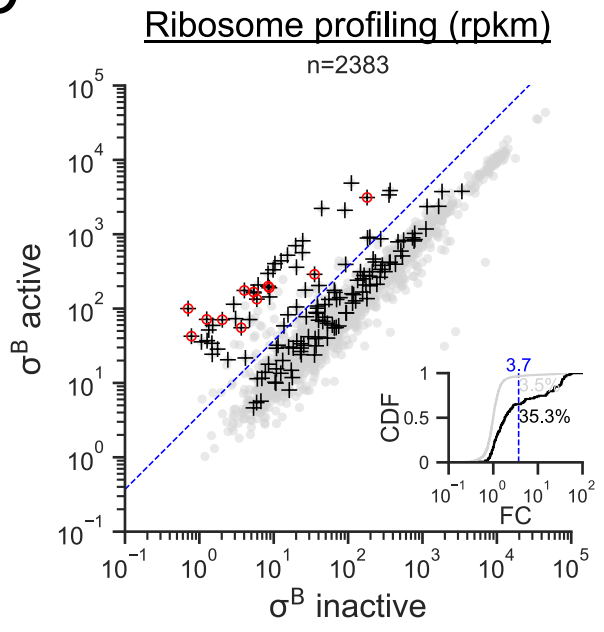

D

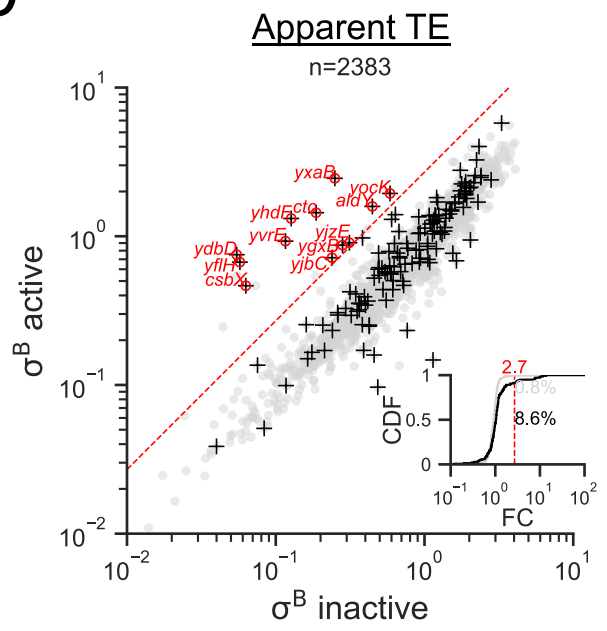




\section{$603 \quad$ Figure 1. $\sigma^{\mathrm{B}}$ activates both transcription and translation.}

604 (A) Models of transcriptional and translational induction for a transcriptional unit consisting of a

605 promoter, coding sequence, and terminator. Stimuli are indicated with lightning bolts and

606 ribosomes are colored in yellow. (B) RNA-seq, (C) ribosome profiling, and (D) apparent

607 translation efficiency measurements from $\sigma^{\mathrm{B}}$ active and inactive conditions. $\sigma^{\mathrm{B}}$ regulon genes are

608 indicated with black crosses $(+)$, and a subset that are translationally activated are highlighted in

609 red. The dashed blue lines mark a 3.7-fold change in expression for visual reference. The dashed

610 red line is an approximate threshold (2.7-fold) separating the population of translationally

611 activated genes from those whose apparent TE does not markedly change. The insets show the

612 cumulative distribution function (CDF) of fold change (FC) across the two conditions in each

613 measurement, with separate CDFs for all genes (gray) and $\sigma^{\mathrm{B}}$ regulon genes (black). The

614 percentage of genes in each group exceeding the chosen thresholds are listed on the right. (E)

615 Contributions of mRNA levels and translation to changes in protein synthesis rate among

616 translationally activated $\sigma^{\mathrm{B}}$ regulon genes. The fold change in protein synthesis rate is indicated

617 by the height of the bars, with the light and dark red regions denoting the respective

618 contributions of mRNA levels and translation, i.e., fold-change in protein synthesis $=($ fold-

619 change in mRNA level $) \times($ fold-change in translation efficiency $)$.

620

621

622

623

624

625 
A
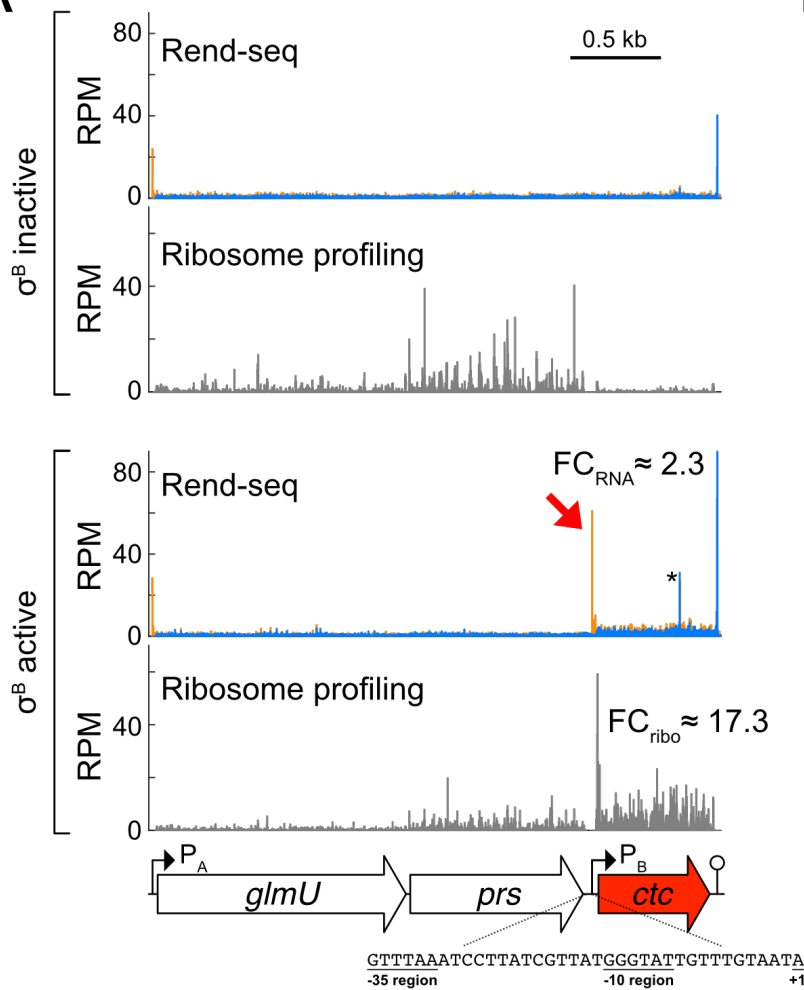

B
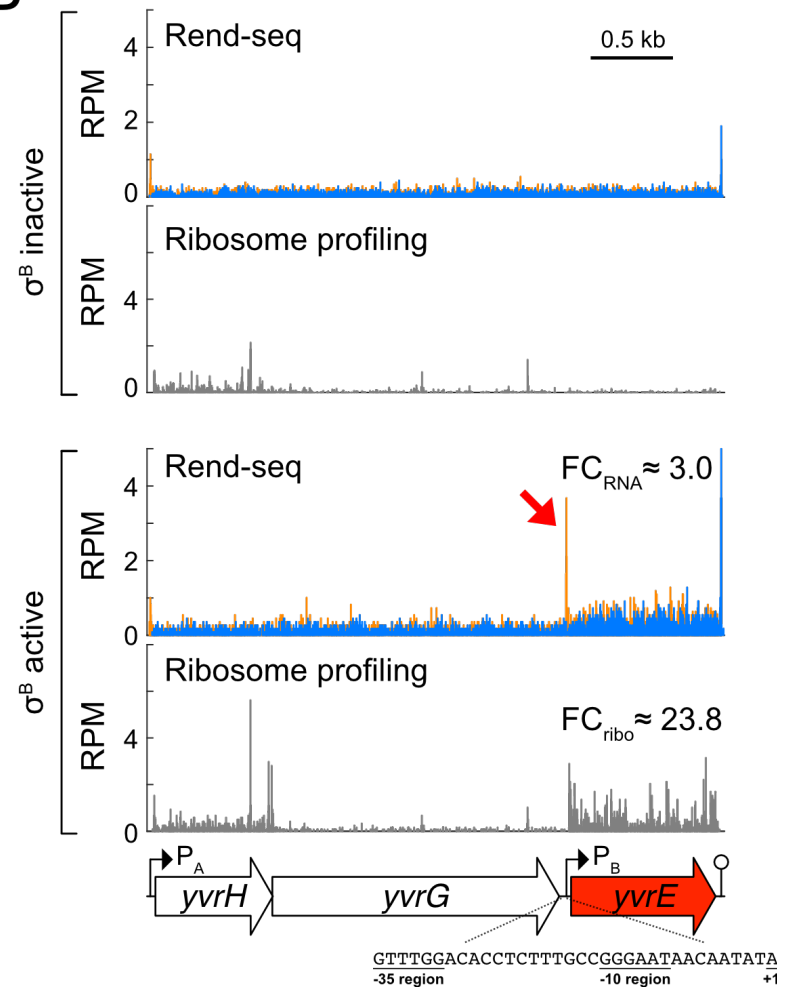

Figure 2. Translationally activated $\sigma^{\mathrm{B}}$ regulon genes display alternative mRNA isoforms.

629 Rend-seq and ribosome profiling data from conditions with inactive/active $\sigma^{\mathrm{B}}$ for the operons containing (A) ctc and $(\mathbf{B})$ yvrE ( $\sigma^{\mathrm{B}}$ regulon genes are highlighted in red). Orange and blue bars

631 represent 5'- and 3'-mapped read counts, respectively, and the black scale bars correspond to 0.5 kb. Fold changes $(\mathrm{FC})$ between $\sigma^{\mathrm{B}}$ active and $\sigma^{\mathrm{B}}$ inactive conditions are shown. Rend-seq 5'

633 ends corresponding to the $\sigma^{\mathrm{B}}$ transcription start sites are marked by red arrows. Putative $\sigma^{\mathrm{B}}$ -

634 dependent promoter sequences are listed for each gene $\left(+1\right.$ corresponds to the 5 , end of the $\sigma^{\mathrm{B}}$ 635 dependent isoform mapped by Rend-seq). The consensus sequences for the -10 and -35 regions 636 of $\sigma^{\mathrm{B}}$-dependent promoters are GTTTaa and GGG(A/T)A(A/T) (Petersohn et al., 1999). For $c t c$

637 specifically, the additional $5^{\prime} / 3^{\prime}$ peak pair $(*)$ in the $\sigma^{\mathrm{B}}$ active condition corresponds to a spurious RNase A cleavage site that likely occurred post-lysis. See also Figures S1 and S2. 
A
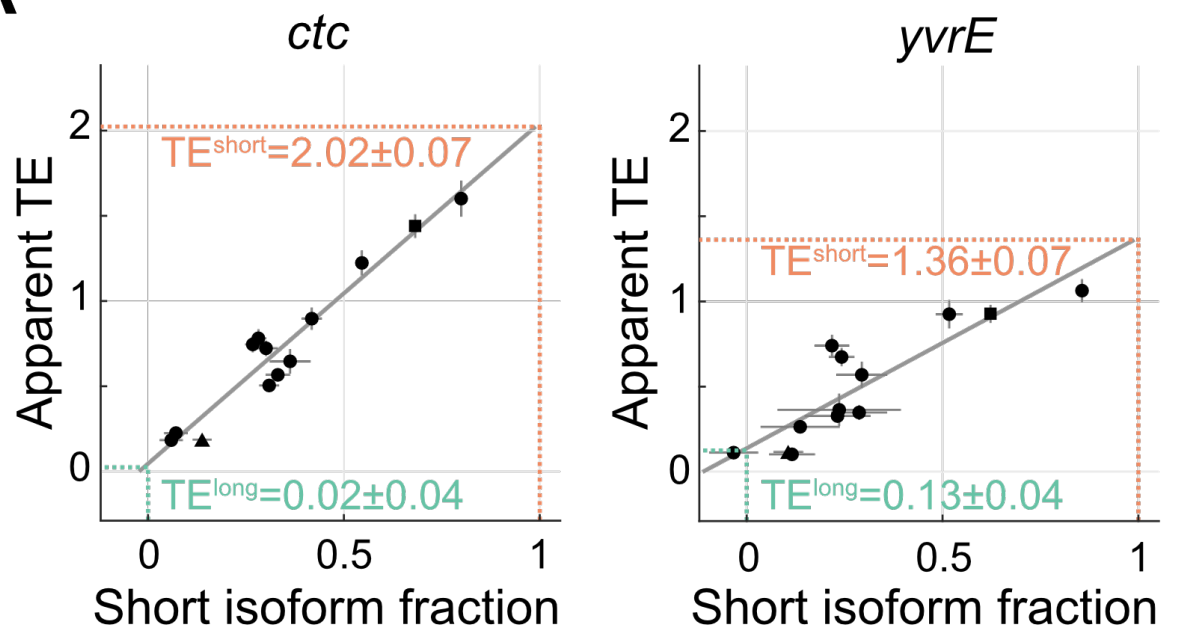

\section{B}

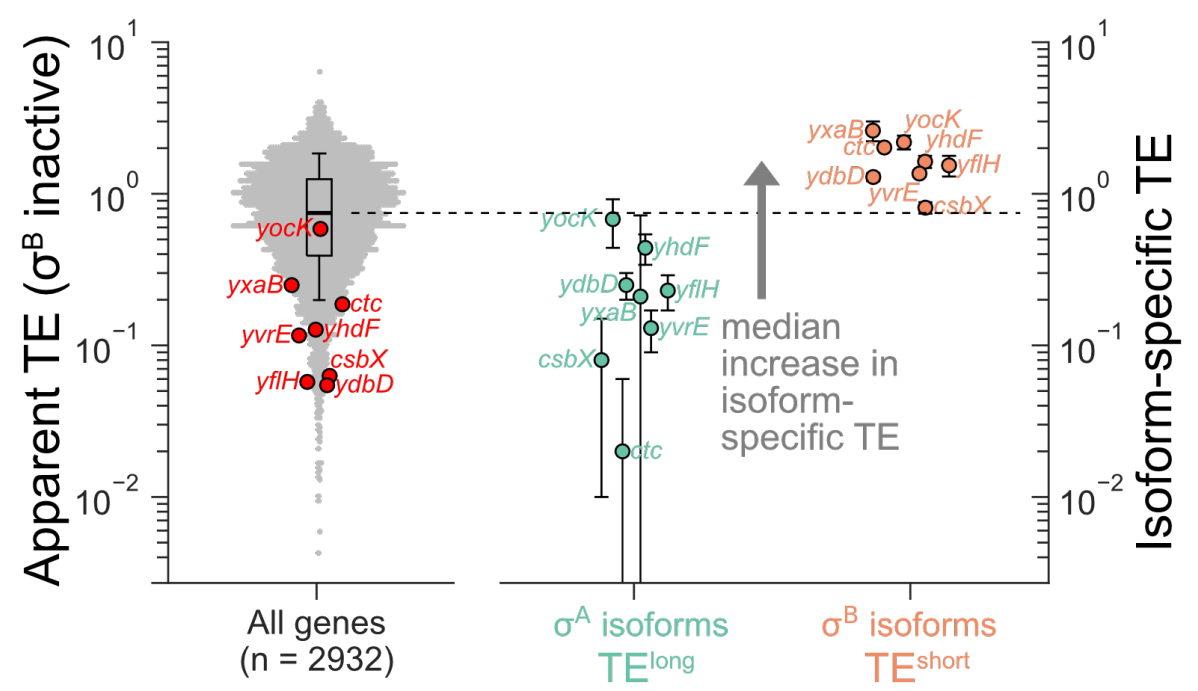

$\mathrm{D}$

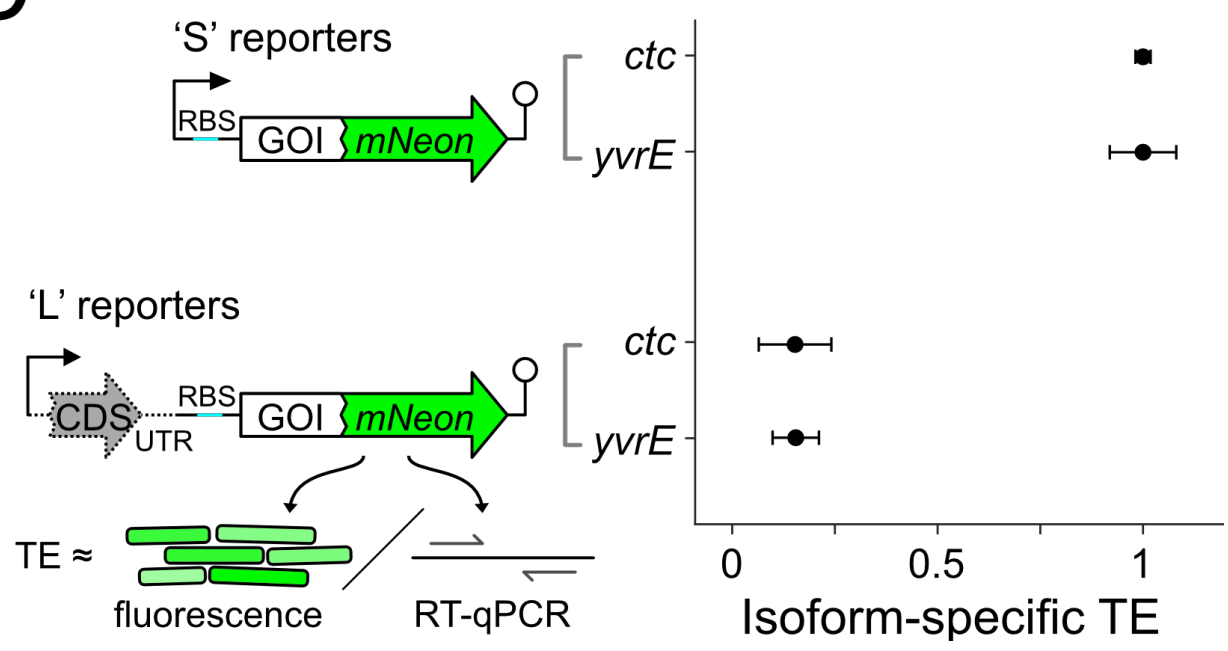




\section{$640 \quad$ Figure 3. $\sigma^{\mathrm{B}}$-dependent mRNA isoforms have elevated TE.}

641 (A) Estimation of the isoform-specific TE for the short, $\sigma^{\mathrm{B}}$-dependent and long, $\sigma^{\mathrm{A}}$-dependent 642 isoforms of $c t c$ and $y v r E$. Each point is an experimental condition which has a different short 643 isoform fraction and correspondingly different apparent TE (conditions shown in Figure 2 are 644 distinctly marked by a triangle and a square for $\sigma^{\mathrm{B}}$ inactive and active, respectively). Error bars

645 correspond to standard deviations from subsampling bootstraps. The gray lines are linear 646 regressions, whereas the dashed lines indicate estimates of isoform-specific TE calculated from

647 the fits (Methods). Estimated isoform-specific TEs and errors (standard deviations) from a

648 bootstrapped linear fit (Methods) are shown. (B) Distribution (beeswarm and boxplot, whiskers 649 corresponding to $10^{\text {th }}$ and $90^{\text {th }}$ percentile) of apparent $\mathrm{TE}$ in $\sigma^{\mathrm{B}}$ inactive conditions.

650 Translationally activated $\sigma^{\mathrm{B}}$-regulon genes (subset from Figure 1 for which isoform-specific TE

651 could be estimated, Methods) are marked (red). (C) Isoform-specific TE values inferred, with

652 error bars as in (A). (D) Fluorescent reporter assay for validating differential TE between

653 isoforms. Protein expression (from fluorescence) and mRNA levels (from reverse-transcription

654 qPCR) were measured for synthetic constructs (left) representing $\sigma^{\mathrm{A}}$-dependent $(\mathrm{L})$ and $\sigma^{\mathrm{B}}$ -

655 dependent isoforms (S). Relative isoform-specific TE (right) was calculated by dividing relative 656 protein expression by relative mRNA levels. Errors bars represent the standard deviation for

657 technical replicates ( $n=3$ for fluorescence, $n=4$ for RT-qPCR). See also Figure S3. 

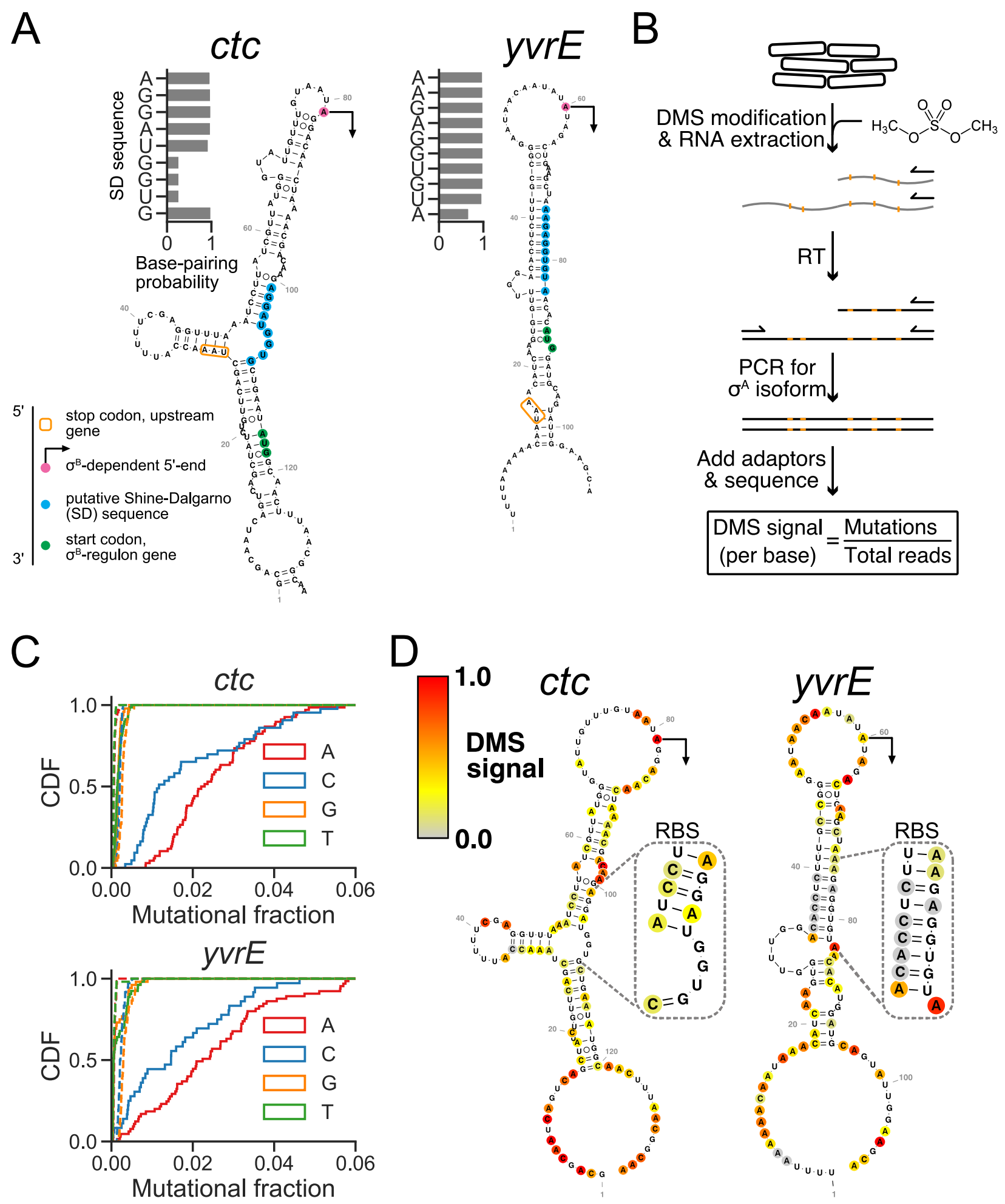


\section{Figure 4. $\sigma^{\mathrm{A}}$-dependent mRNA isoforms have extended secondary structures in vivo.}

668 (A) Minimum free energy (MFE) structures of the $\sigma^{\mathrm{A}}$-dependent isoforms of $c t c$ and $y v r E$ near

669 the ribosome binding site. The transcription start sites of $\sigma^{\mathrm{B}}$-dependent isoforms (indicated with

670 arrows), putative Shine-Dalgarno (SD) sequences, and start codons are highlighted in magenta,

671 blue, and green, respectively. The stop codon of the upstream gene in the operon is indicated

672 with an orange box. Computationally-determined base-pairing probabilities for individual bases

673 in the SD sequences are shown beside each structure. (B) DMS-MaPseq workflow for in vivo

674 RNA structure determination of $\sigma^{\mathrm{A}}$-dependent isoforms. (C) Cumulative distributions of the per-

675 base mutational fractions for the $\sigma^{\mathrm{A}}$-dependent isoforms of $c t c$ and $y v r E$. Solid and dashed lines

676 indicate conditions with and without DMS treatment. (D) DMS-constrained MFE structures of

677 representative transcripts for $\sigma^{\mathrm{A}}$-dependent isoforms of $c t c$ and $y v r E$ colored by normalized

678 DMS-MaPseq mutation rate (DMS signal), where values correspond to increased base

679 accessibility. Structured regions containing putative SD sequences are magnified.

680

681

682

683

684

685

686 

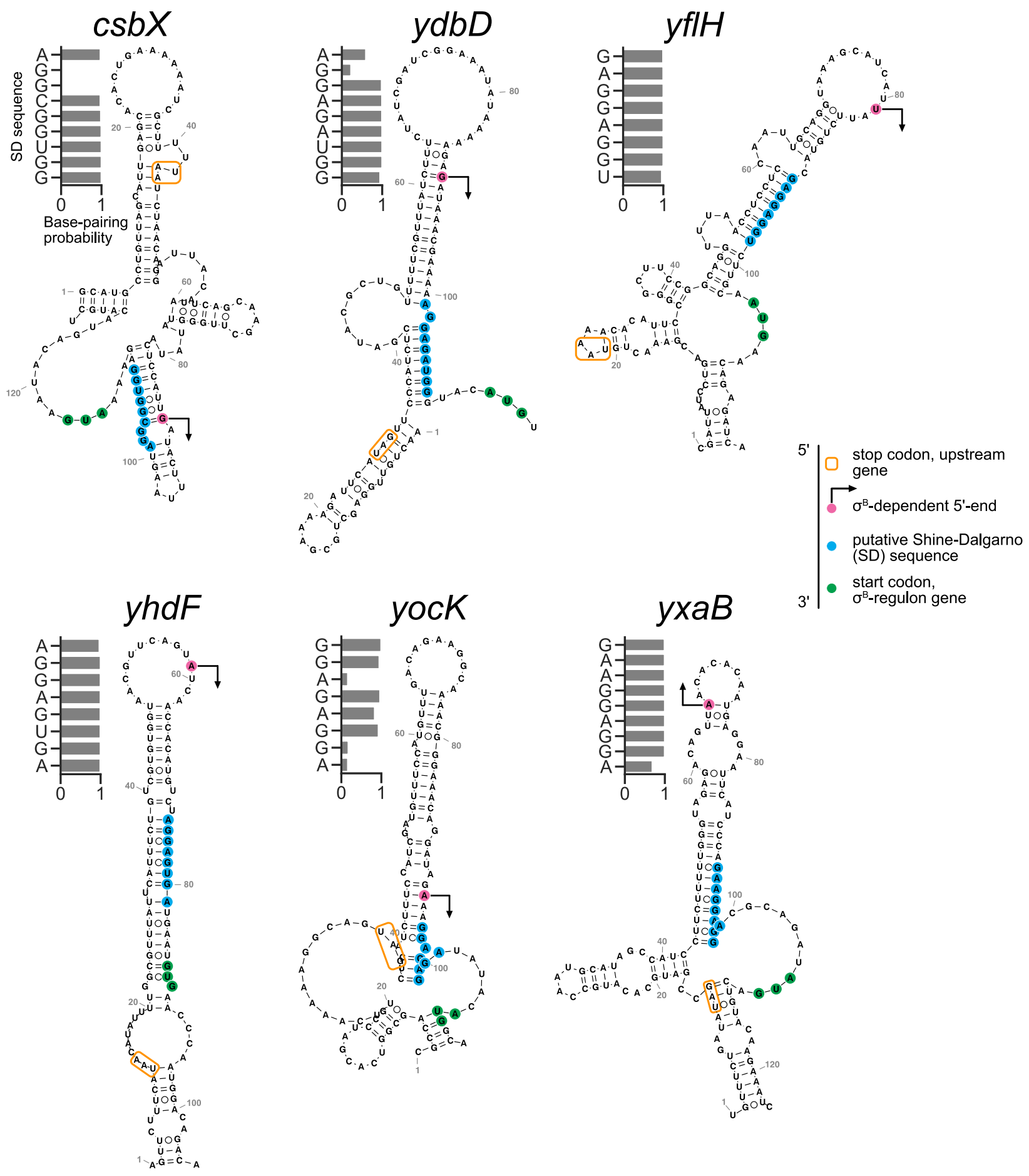


\section{Figure 5. Long-range mRNA secondary structures in $\sigma^{\mathbf{A}}$-dependent isoforms sequester}

695 sequence elements necessary for translation.

696 MFE structures of transcripts for $\sigma^{\mathrm{A}}$-dependent isoforms of other translationally activated $\sigma^{\mathrm{B}}$

697 regulon genes. The transcription start sites of $\sigma^{\mathrm{B}}$-dependent isoforms (indicated with arrows),

698 putative Shine-Dalgarno (SD) sequences, and start codons are highlighted in magenta, blue, and

699 green, respectively. The stop codon of the upstream gene in the operon is indicated with an

700 orange box. Computationally-determined base-pairing probabilities for individual bases in the

701 SD sequences are shown beside each structure.

702

703

704

705

706

707

708

709

710

711

712

713

714

715

716 


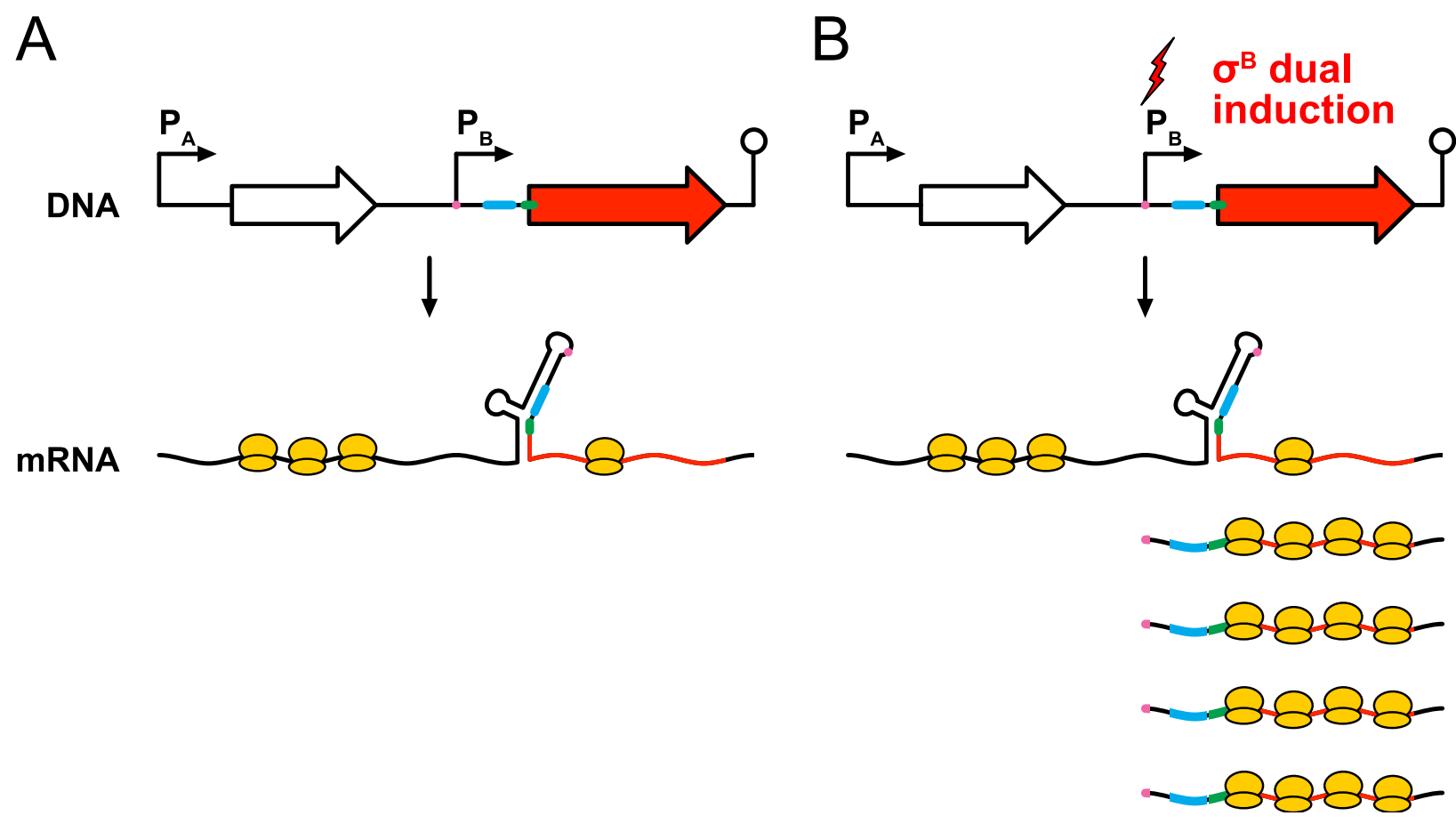

719 Figure 6. Model for $\sigma^{\mathrm{B}}$-dependent translational activation.

720 Schematic of a polycistronic operon containing a $\sigma^{\mathrm{A}}$-dependent promoter $\left(\mathrm{P}_{\mathrm{A}}\right), \sigma^{\mathrm{B}}$-dependent

721 promoter $\left(\mathrm{P}_{\mathrm{B}}\right)$, coding sequences, and a terminator. $(\mathrm{A})$ In the absence of $\sigma^{\mathrm{B}}$, transcription from

$722 \quad \mathrm{P}_{\mathrm{A}}$ produces a polycistronic mRNA molecule containing secondary structures that translationally

723 repress the $\sigma^{\mathrm{B}}$-dependent open reading frame (red) by sequestering its Shine-Dalgarno sequence

724 (blue) and start codon (green). (B) $\mathrm{P}_{\mathrm{B}}$ becomes transcriptionally active upon $\sigma^{\mathrm{B}}$ induction,

725 generating an mRNA isoform with an alternative transcription start site (magenta). Without the

726 sequences necessary to form stable secondary structures, these transcripts can recruit ribosomes

727 more efficiently to facilitate greater protein expression. 